ANNALES

POLONICI MATHEMATICI

LXXIX.2 (2002)

\title{
Periodic solutions of dissipative dynamical systems with singular potential and $p$-Laplacian
}

\author{
by Bing LiU (Wuhan)
}

\begin{abstract}
By using the topological degree theory and some analytic methods, we consider the periodic boundary value problem for the singular dissipative dynamical systems with $p$-Laplacian: $\left(\phi_{p}\left(x^{\prime}\right)\right)^{\prime}+\frac{d}{d t} \operatorname{grad} F(x)+\operatorname{grad} G(x)=e(t), x(0)=x(T)$, $x^{\prime}(0)=x^{\prime}(T)$. Sufficient conditions to guarantee the existence of solutions are obtained under no restriction on the damping forces $\frac{d}{d t} \operatorname{grad} F(x)$.
\end{abstract}

1. Introduction. Consider the periodic boundary value problem for the singular dissipative dynamical system with $p$-Laplacian

$$
\begin{gathered}
\left(\phi_{p}\left(x^{\prime}\right)\right)^{\prime}+\frac{d}{d t} \operatorname{grad} F(x)+\operatorname{grad} G(x)=e(t), \\
x(0)=x(T), \quad x^{\prime}(0)=x^{\prime}(T),
\end{gathered}
$$

where $F \in C^{2}\left(\mathbb{R}^{n}, \mathbb{R}\right), G \in C^{1}\left(\mathbb{R}^{n} \backslash\{0\}, \mathbb{R}\right), e \in L^{1}\left([0, T], \mathbb{R}^{n}\right), p>1$ is a fixed constant, and $\phi_{p}: \mathbb{R}^{n} \rightarrow \mathbb{R}^{n}$ is the mapping defined by

$$
\phi_{p}(x)=\phi_{p}\left(x_{1}, \ldots, x_{n}\right)=\left(\left|x_{1}\right|^{p-2} x_{1}, \ldots,\left|x_{n}\right|^{p-2} x_{n}\right) .
$$

Then $\phi_{p}$ is a homeomorphism of $\mathbb{R}^{n}$ with inverse $\phi_{q}(1 / q+1 / p=1)$.

Roughly speaking, the potential $G(x)$ is singular (at 0 ) in the sense that $G(x)$ becomes infinite when $x$ tends to 0 . From the physical meaning of (1), the singular potential $G(x)$ is said to be of attractive type (repulsive type, respectively) if $G(x) \rightarrow-\infty(G(x) \rightarrow \infty$, respectively) as $x \rightarrow 0$; see Ambrosetti [1].

By a solution $x$ of problem (1), (2), we mean that $x \in W^{2, p}\left([0, T], \mathbb{R}^{n}\right)$, $\phi_{p}\left(x^{\prime}\right)$ is absolutely continuous on $[0, T], x(t) \neq 0$ for all $t \in[0, T]$ and $x$ satisfies the boundary condition (2) and equation (1) for a.e. $t \in[0, T]$.

When $p=2$ or $\phi_{p}(x)=x$, and no damping is present in (1), the above boundary value problem has variational structure and can be handled by critical point theory. In [3], Gordon introduced a strong force condition on

2000 Mathematics Subject Classification: Primary 34C15, 34C25.

Key words and phrases: singular potential, dissipative system, $p$-Laplacian. 
$G(x)$ at 0 to get some compactness for the corresponding action functionals. We refer to Ambrosetti [1] for attractive case and to Coti Zelati [2] and Solimini $[9]$ for the repulsive case.

When damping is present in (1), and $p=2$ or $\phi_{p}(x)=x$, we can use the Leray-Schauder degree or the coincidence degree. For one-dimensional systems, we refer to $[5,6,8,12]$. For higher dimensional systems, Habets and Sanchez [4] introduced a different strong force condition on $G(x)$ at the origin from the viewpoint of degree theory. Zhang [10] obtained an existence result under no restriction on the damping forces $\frac{d}{d t} \operatorname{grad} F(x)$.

In the present paper, motivated by [10], we are also interested in higher dimensional systems. We establish the existence results for repulsive type and attractive type and for any damping forces, without imposing more conditions on the singular potential $G(x)$. Moreover, the general exponent $p$ is allowed, and our results seem to be new even if $p=2$.

The notation we use is mostly standard. We denote the Euclidean inner product in $\mathbb{R}^{n}$ by $\langle\cdot, \cdot\rangle$, and the $l^{p}$-norm in $\mathbb{R}^{n}$ by $|\cdot|$, i.e.

$$
|x|=\left|\left(x_{1}, \ldots, x_{n}\right)\right|=\left(\sum_{i=1}^{n}\left|x_{i}\right|^{p}\right)^{1 / p} .
$$

The corresponding $L^{p}$-norm in $L^{p}\left([0, T], \mathbb{R}^{n}\right)$ is defined by

$$
\|x\|_{p}=\left(\sum_{i=1}^{n} \int_{0}^{1}\left|x_{i}(t)\right|^{p} d t\right)^{1 / p}=\left(\sum_{i=1}^{n}\left\|x_{i}\right\|_{p}^{p}\right)^{1 / p}
$$

where $x_{i} \in L^{p}([0, T], \mathbb{R}),\left\|x_{i}\right\|_{p}=\left(\int_{0}^{1}\left|x_{i}(t)\right|^{p} d t\right)^{1 / p}(i=1, \ldots, n)$. The $L^{\infty}$ norm in $C\left([0, T], \mathbb{R}^{n}\right)$ is

$$
\|x\|_{\infty}=\max _{1 \leq i \leq n}\left\|x_{i}\right\|_{\infty}
$$

where $\left\|x_{i}\right\|_{\infty}=\sup _{t \in[0, T]}\left|x_{i}(t)\right|(i=1, \ldots, n)$. Also, for $n \geq 1$, we write $C^{1}=C^{1}\left([0, T], \mathbb{R}^{n}\right), C_{T}^{1}=\left\{x \in C^{1}: x(0)=x(T), x^{\prime}(0)=x^{\prime}(T)\right\}$.

2. Some lemmas. To discuss the existence of solutions for the periodic boundary value problem (1), (2), we introduce some lemmas.

Let $\phi: \mathbb{R}^{n} \rightarrow \mathbb{R}^{n}$ be a continuous function which satisfies the following two conditions (see $[7]$ ):

$\left(\mathrm{H}_{1}\right) \quad$ For any $x, y \in \mathbb{R}^{n}, x \neq y$, one has

$$
\langle\phi(x)-\phi(y), x-y\rangle>0 .
$$

$\left(\mathrm{H}_{2}\right) \quad$ There exists a function $\beta:[0, \infty) \rightarrow[0, \infty)$ such that $\lim _{s \rightarrow \infty} \beta(s)$ $=\infty$ and

$$
\langle\phi(x), x\rangle \geq \beta(|x|)|x| \quad \text { for all } x \in \mathbb{R}^{n} .
$$


Clearly, under these two conditions $\phi$ is a homeomorphism of $\mathbb{R}^{n}$ onto $\mathbb{R}^{n}$, and $\lim _{|s| \rightarrow \infty}\left|\phi^{-1}(s)\right|=\infty$ (see [7]). We notice that, in [7], the $|\cdot|$ of $\left(\mathrm{H}_{2}\right)$ denotes the Euclidean norm in $\mathbb{R}^{n}$, while here it is the $l^{p}$-norm in $\mathbb{R}^{n}$. However, it is well known that any two norms on $\mathbb{R}^{n}$ are equivalent, so the above condition $\left(\mathrm{H}_{2}\right)$ and the condition $\left(\mathrm{H}_{2}\right)$ of [7] are equivalent.

First, consider the periodic boundary value problem

$$
\begin{aligned}
& \left(\phi\left(x^{\prime}\right)\right)^{\prime}=f\left(t, x, x^{\prime}\right), \\
& x(0)=x(T), \quad x^{\prime}(0)=x^{\prime}(T),
\end{aligned}
$$

where $\phi: \mathbb{R}^{n} \rightarrow \mathbb{R}^{n}$ satisfies conditions $\left(\mathrm{H}_{1}\right),\left(\mathrm{H}_{2}\right)$, and $f:[0, T] \times \mathbb{R}^{n} \times \mathbb{R}^{n} \rightarrow$ $\mathbb{R}^{n}$ is a Carathéodory function. Then we have the following lemma (see Theorem 3.1 of [7]).

Lemma 1 (see [7]). Assume that $\Omega$ is an open bounded set in $C_{T}^{1}$ such that the following conditions hold:

(i) For each $\lambda \in(0,1)$, the problem

$$
\left\{\begin{array}{l}
\left(\phi\left(x^{\prime}\right)\right)^{\prime}=\lambda f\left(t, x, x^{\prime}\right), \\
x(0)=x(T), \quad x^{\prime}(0)=x^{\prime}(T),
\end{array}\right.
$$

has no solution on $\partial \Omega$.

(ii) The equation

$$
\widetilde{F}(d)=\frac{1}{T} \int_{0}^{T} f(t, d, 0) d t=0
$$

has no solution on $\partial \Omega \cap \mathbb{R}^{n}$.

(iii) The Brouwer degree

$$
\operatorname{deg}_{\mathrm{B}}\left[\widetilde{F}, \Omega \cap \mathbb{R}^{n}, 0\right] \neq 0 .
$$

Then problem (4), (5) has a solution in $\bar{\Omega}$.

Next, let $W^{1, p}\left([0, T], \mathbb{R}^{n}\right)$ be the Sobolev space. We need the following inequality (see Lemma 3 of [11]):

Lemma 2 (see [11]). Let $u \in W^{1, p}\left([0, T], \mathbb{R}^{n}\right), u(0)=u(T)=0$. Then

$$
\|u\|_{p} \leq \frac{T}{\pi_{p}}\left\|u^{\prime}\right\|_{p}
$$

where

$$
\pi_{p}=2 \int_{0}^{(p-1)^{1 / p}} \frac{d s}{\left(1-\frac{s^{p}}{p-1}\right)^{1 / p}}=\frac{2 \pi(p-1)^{1 / p}}{p \sin \left(\frac{\pi}{p}\right)} .
$$

3. Main results. In this section, we establish the existence results for the repulsive type and attractive type. 
Lemma 3. Assume that $\phi_{p}: \mathbb{R}^{n} \rightarrow \mathbb{R}^{n}$ is given by (3). Then $\phi_{p}$ satisfies conditions $\left(\mathrm{H}_{1}\right),\left(\mathrm{H}_{2}\right)$.

Proof. Let $x, y \in \mathbb{R}^{n}, x \neq y$. From the inequality

$$
\begin{aligned}
\left\langle\phi_{p}(x)-\phi_{p}(y), x-y\right\rangle & =\sum_{i=1}^{n}\left[\left|x_{i}\right|^{p}-x_{i} y_{i}\left(\left|x_{i}\right|^{p-2}+\left|y_{i}\right|^{p-2}\right)+\left|y_{i}\right|^{p}\right] \\
& \geq \sum_{i=1}^{n}\left[\left|x_{i}\right|^{p}-\left|x_{i}\right| \cdot\left|y_{i}\right|\left(\left|x_{i}\right|^{p-2}+\left|y_{i}\right|^{p-2}\right)+\left|y_{i}\right|^{p}\right] \\
& =\sum_{i=1}^{n}\left[\left|x_{i}\right|^{p-1}-\left|y_{i}\right|^{p-1}\right]\left[\left|x_{i}\right|-\left|y_{i}\right|\right] \geq 0
\end{aligned}
$$

it follows immediately that

$$
\left\langle\phi_{p}(x)-\phi_{p}(y), x-y\right\rangle=0 \Rightarrow x=y
$$

and thus $\left(\mathrm{H}_{1}\right)$ holds. Also, from

$$
\left\langle\phi_{p}(x), x\right\rangle=\sum_{i=1}^{n}\left|x_{i}\right|^{p}=|x|^{p}=|x|^{p-1}|x|,
$$

$\left(\mathrm{H}_{2}\right)$ follows.

First, for the repulsive type, we have the following result.

THEOREM 1. Assume that the following conditions hold:

$\left(\mathrm{R}_{1}\right) \quad \lim _{x \rightarrow 0}\langle x, \operatorname{grad} G(x)\rangle=-\infty$.

$\left(\mathrm{R}_{2}\right) \quad$ Habets-Sanchez's strong force condition at 0 (see [4]): There exists $\varphi \in C^{1}\left(\mathbb{R}^{n} \backslash\{0\}, \mathbb{R}\right)$ such that (i) $\lim _{x \rightarrow 0} \varphi(x)=\infty$; and (ii) $|\operatorname{grad} \varphi(x)|^{q} \leq|\langle x, \operatorname{grad} G(x)\rangle|+c_{0}$ if $0<|x| \ll 1$, where $c_{0}$ is a positive constant and $1 / q+1 / p=1$.

$\left(\mathrm{R}_{3}\right) \quad$ There exist nonnegative constants $a, b$ and $c$ such that

$$
\langle x, \operatorname{grad} G(x)\rangle \leq a|x|^{p}+c, \quad \forall x \in \mathbb{R}^{n} \backslash\{0\} .
$$

$\left(\mathrm{R}_{4}\right) \quad$ There exists a constant $M>0$ such that if $\left|x_{i}\right|>M(i=1, \ldots, n)$, then

$$
x_{i}\left(\frac{\partial G}{\partial x_{i}}-\bar{e}_{i}\right)<0 \quad \text { for all } i \in\{1, \ldots, n\}
$$

where $\bar{e}_{i}=T^{-1} \int_{0}^{T} e_{i}(t) d t(i=1, \ldots, n)$.

Then the periodic boundary value problem (1), (2) has at least one solution provided

$$
a<\left(\pi_{p} / T\right)^{p}
$$

where $\pi_{p}$ is defined by (6). 
Proof. To apply Lemma 1, we first consider the auxiliary equation

$$
\left\{\begin{array}{l}
\left(\phi_{p}\left(x^{\prime}\right)\right)^{\prime}=\lambda\left[e(t)-\frac{d}{d t} \operatorname{grad} F(x)-\operatorname{grad} G(x)\right], \quad \lambda \in(0,1), \\
x(0)=x(T), \quad x^{\prime}(0)=x^{\prime}(T) .
\end{array}\right.
$$

Set

$$
\Omega_{1}=\left\{x \in W^{2, p}\left([0, T], \mathbb{R}^{n}\right): x \text { satisfies }(7)\right\} .
$$

We shall show that there exist positive constants $M_{i}(i=1,2,3,4)$ such that, for any $x \in \Omega_{1}$,

$$
\left\|x^{\prime}\right\|_{p}<M_{1}, \quad M_{2}<\|x\|_{\infty}<M_{3}, \quad\left\|x^{\prime}\right\|_{\infty}<M_{4} .
$$

In fact, for $x \in \Omega_{1}$, from (7), one has

$$
\left(\phi_{p}\left(x^{\prime}\right)\right)^{\prime}=\lambda\left[e(t)-\frac{d}{d t} \operatorname{grad} F(x)-\operatorname{grad} G(x)\right] .
$$

Since $x(0)=x(T), x^{\prime}(0)=x^{\prime}(T)$, we have

$$
-\left\|x^{\prime}\right\|_{p}^{p}=\int_{0}^{T}\left\langle x,\left(\phi_{p}\left(x^{\prime}\right)\right)^{\prime}\right\rangle d t
$$

and

$$
\left\langle x, \frac{d}{d t} \operatorname{grad} F(x)\right\rangle=\frac{d}{d t}\langle x, \operatorname{grad} F(x)\rangle-\left\langle x^{\prime}, \operatorname{grad} F(x)\right\rangle .
$$

Thus considering the inner product of $(9)$ with $x$, from $\left(\mathrm{R}_{3}\right)$ we deduce that

$$
\begin{aligned}
\left\|x^{\prime}\right\|_{p}^{p} & =\lambda \int_{0}^{T}\langle x, \operatorname{grad} G(x)\rangle d t-\lambda \int_{0}^{T}\langle x, e(t)\rangle d t \\
& \leq \int_{0}^{T}\left[a|x(t)|^{p}+c\right] d t+\int_{0}^{T}|x(t)| \cdot|e(t)| d t \\
& \leq a\|x\|_{p}^{p}+\|e\|_{1}\|x\|_{\infty}+c T .
\end{aligned}
$$

Again from (9), we obtain

$$
\begin{aligned}
0=\int_{0}^{T}\left(\phi_{p}\left(x^{\prime}\right)\right)^{\prime} d t & =\lambda \int_{0}^{T}\left[e(t)-\frac{d}{d t} \operatorname{grad} F(x)-\operatorname{grad} G(x)\right] d t \\
& =\lambda \int_{0}^{T}[e(t)-\operatorname{grad} G(x)] d t
\end{aligned}
$$

and thus

$$
\frac{1}{T} \int_{0}^{T} \operatorname{grad} G(x) d t=\frac{1}{T} \int_{0}^{T} e(t) d t
$$


that is,

$$
\frac{1}{T} \int_{0}^{T} \frac{\partial G}{\partial x_{i}} d t=\bar{e}_{i}, \quad i=1, \ldots, n .
$$

So in view of $\left(\mathrm{R}_{4}\right)$, for any $i \in\{1, \ldots, n\}$, there exists $t_{i} \in[0, T]$ such that $\left|x_{i}\left(t_{i}\right)\right| \leq M$. Thus for any $i \in\{1, \ldots, n\}$,

$$
\begin{aligned}
\left|x_{i}(t)\right| & =\left|x_{i}\left(t_{i}\right)+\int_{t_{i}}^{t} x_{i}^{\prime}(s) d s\right| \leq M+\int_{0}^{T}\left|x_{i}^{\prime}(s)\right| d s \\
& \leq M+T^{1 / q}\left(\int_{0}^{T}\left|x_{i}^{\prime}(s)\right|^{p} d t\right)^{1 / p} \leq M+T^{1 / q}\left(\sum_{i=1}^{n} \int_{0}^{T}\left|x_{i}^{\prime}(s)\right|^{p} d s\right)^{1 / p} \\
& =M+T^{1 / q}\left\|x^{\prime}\right\|_{p}
\end{aligned}
$$

where $1 / q+1 / p=1$. From this inequality, we have $\left\|x_{i}\right\|_{\infty} \leq M+T^{1 / q}\left\|x^{\prime}\right\|_{p}$ so

$$
\|x\|_{\infty} \leq M+T^{1 / q}\left\|x^{\prime}\right\|_{p} .
$$

Again for each $i \in\{1, \ldots, n\}$, set

$$
y_{i}(t)= \begin{cases}x_{i}\left(t+t_{i}-T\right)-x_{i}\left(t_{i}\right), & T-t_{i} \leq t \leq T \\ x_{i}\left(t+t_{i}\right)-x_{i}\left(t_{i}\right), & 0 \leq t \leq T-t_{i}\end{cases}
$$

Then $y_{i}(0)=y_{i}(T)=0$, hence $y(0)=y(T)=0$. Since $x \in W^{2, p}\left([0, T], \mathbb{R}^{n}\right)$, we see that $y \in W^{2, p}\left([0, T], \mathbb{R}^{n}\right)$, which implies $y \in W^{1, p}\left([0, T], \mathbb{R}^{n}\right)$. Therefore, from Lemma 2 ,

$$
\|y\|_{p} \leq \frac{T}{\pi_{p}}\left\|y^{\prime}\right\|_{p}
$$

For each $i \in\{1, \ldots, n\}$, we have

$$
\begin{aligned}
\left\|y_{i}+x_{i}\left(t_{i}\right)\right\|_{p}^{p} & =\int_{0}^{T-t_{i}}\left|x_{i}\left(t+t_{i}\right)\right|^{p} d t+\int_{T-t_{i}}^{T}\left|x\left(t+t_{i}-T\right)\right|^{p} d t \\
& =\int_{t_{i}}^{T}\left|x_{i}(t)\right|^{p} d t+\int_{0}^{t_{i}}\left|x_{i}(t)\right|^{p} d t=\left\|x_{i}\right\|_{p}^{p} .
\end{aligned}
$$

For any $\varepsilon \in\left(0, a^{-1}\left(\pi_{p} / T\right)^{p}-1\right)$, in view of the Minkowski inequality, $p>1$ and $\left|x_{i}\left(t_{i}\right)\right| \leq M$, one has

$$
\begin{aligned}
\left\|x_{i}\right\|_{p}^{p} & =\left\|y_{i}+x_{i}\left(t_{i}\right)\right\|_{p}^{p} \leq\left(\left\|y_{i}\right\|_{p}+\left\|x_{i}\left(t_{i}\right)\right\|_{p}\right)^{p} \leq\left(\left\|y_{i}\right\|_{p}+T^{1 / p} M\right)^{p} \\
& \leq(1+\varepsilon)\left\|y_{i}\right\|_{p}^{p}+\text { const }
\end{aligned}
$$


which implies

$$
\|x\|_{p}^{p}=\sum_{i=1}^{n}\left\|x_{i}\right\|_{p}^{p} \leq(1+\varepsilon)\|y\|_{p}^{p}+\text { const. }
$$

Noticing $y_{i}^{\prime}=x_{i}^{\prime}(i=1, \ldots, n)$, which implies $\left\|y^{\prime}\right\|_{p}=\left\|x^{\prime}\right\|_{p}$, from (12), (13) we obtain

$$
\|x\|_{p}^{p} \leq(1+\varepsilon)\left(\frac{T}{\pi_{p}}\right)^{p}\left\|x^{\prime}\right\|_{p}^{p}+\text { const. }
$$

Hence from (10), (11), (14), one has

$$
\left\|x^{\prime}\right\|_{p}^{p} \leq a(1+\varepsilon)\left(\frac{T}{\pi_{p}}\right)^{p}\left\|x^{\prime}\right\|_{p}^{p}+T^{1 / q}\|e\|_{1}\left\|x^{\prime}\right\|_{p}+\text { const. }
$$

As $\varepsilon \in\left(0, a^{-1}\left(\pi_{p} / T\right)^{p}-1\right)$, we have $a(1+\varepsilon)\left(T / \pi_{p}\right)^{p}<1$, and thus from the above inequality, there exists a constant $M_{1}>0$ such that

$$
\left\|x^{\prime}\right\|_{p}<M_{1} \text {. }
$$

Taking

$$
M_{3}>M+T^{1 / q} M_{1},
$$

from (11), (15), (16), one has

$$
\|x\|_{\infty}<M_{3}
$$

Now in view of conditions $\left(\mathrm{R}_{1}\right),\left(\mathrm{R}_{2}\right)$, proceeding as in the proof of Lemma 6 of [4], a lower bound for $\min \{|x(t)|: t \in[0, T]\}$ follows from the proof by noticing the estimates (15), (17). Namely, there is some $L>0$ such that

$$
\min \{|x(t)|: t \in[0, T]\}>L .
$$

Since $\|x\|_{\infty}=\max _{1 \leq i \leq n}\left\|x_{i}\right\|_{\infty}$ where $\left\|x_{i}\right\|_{\infty}=\sup _{t \in[0, T]}\left|x_{i}(t)\right|$, we have

$$
\begin{aligned}
n^{1 / p}\|x\|_{\infty} & \geq\left(\sum_{i=1}^{n}\left\|x_{i}\right\|_{\infty}^{p}\right)^{1 / p}=\left[\sum_{i=1}^{n}\left(\sup _{t \in[0, T]}\left|x_{i}(t)\right|\right)^{p}\right]^{1 / p} \\
& \geq\left[\sum_{i=1}^{n}\left|x_{i}(t)\right|^{p}\right]^{1 / p}=|x(t)| \geq \min _{t \in[0, T]}|x(t)|>L .
\end{aligned}
$$

Again from condition $\left(\mathrm{R}_{1}\right)$, there exists a small enough $M_{2} \in\left(0, n^{-1 / p} L\right)$ such that, for any $d \in \mathbb{R}^{n}$ with $\|d\|_{\infty} \leq M_{2}$, one has

$$
\left|\left\langle d,\left.\operatorname{grad} G(x)\right|_{x=d}\right\rangle\right|>M_{2} \sum_{i=1}^{n}\left|\bar{e}_{i}\right| .
$$

Since $M_{2}<n^{-1 / p} L$, from (18) we have

$$
\|x\|_{\infty}>M_{2} \text {. }
$$


Again by (17), (18), for any $t \in[0, T]$, one has $L<|x(t)| \leq n^{1 / p}\|x\|_{\infty} \leq$ $n^{1 / p} M_{3}$. Thus since $F \in C^{2}\left(\mathbb{R}^{n}, \mathbb{R}\right)$ and $G \in C^{1}\left(\mathbb{R}^{n} \backslash\{0\}, \mathbb{R}\right)$, there exist constants $L_{1}, L_{2}>0$ such that $\left|\partial^{2} F(x) / \partial x^{2}\right| \leq L_{1}$ and $|\operatorname{grad} G(x)| \leq L_{2}$. Now we use (9) to get

$$
\begin{aligned}
\int_{0}^{T}\left|\left(\phi_{p}\left(x^{\prime}\right)\right)^{\prime}\right| d t & =\lambda \int_{0}^{T}\left|e(t)-\frac{d}{d t} \operatorname{grad} F(x)-\operatorname{grad} G(x)\right| d t \\
& \leq \int_{0}^{T}|e(t)| d t+\int_{0}^{T}\left|\frac{d}{d t} \operatorname{grad} F(x)\right| d t+\int_{0}^{T}|\operatorname{grad} G(x)| d t \\
& =\int_{0}^{T}|e(t)| d t+\int_{0}^{t}\left|\frac{\partial^{2} F}{\partial x^{2}}\right|\left|x^{\prime}\right| d t+\int_{0}^{T}|\operatorname{grad} G(x)| d t \\
& \leq \int_{0}^{T}|e(t)| d t+L_{1} \int_{0}^{T}\left|x^{\prime}\right| d t+L_{2} T \\
& \leq\|e\|_{1}+L_{1} T^{1 / q}\left\|x^{\prime}\right\|_{p}+L_{2} T .
\end{aligned}
$$

From (15), (21), there exists a constant $L_{3}>\|e\|_{1}+T^{1 / q} L_{1} M_{1}+L_{2} T$ such that

$$
\int_{0}^{T}\left|\left(\phi_{p}\left(x^{\prime}\right)\right)^{\prime}\right| d t<L_{3}
$$

On the other hand, for any $i \in\{1, \ldots, n\}$, as $x_{i}(0)=x_{i}(T)$, there exists $t_{i}^{*} \in(0, T)$ such that $x_{i}^{\prime}\left(t_{i}^{*}\right)=0$; thus from (22) we get

$$
\left|\phi_{p}\left(x_{i}^{\prime}(t)\right)\right|=\left|\phi_{p}\left(x_{i}^{\prime}(t)\right)-\phi_{p}\left(x_{i}^{\prime}\left(t_{i}^{*}\right)\right)\right|=\left|\int_{t_{i}^{*}}^{t}\left(\phi_{p}\left(x_{i}^{\prime}(s)\right)\right)^{\prime} d s\right| \leq L_{3} .
$$

So $\left|x_{i}^{\prime}(t)\right| \leq \phi_{q}\left(L_{3}\right)$ for all $i \in\{1, \ldots, n\}$ and $t \in[0, T]$. Hence there exists a constant $M_{4}>\phi_{q}\left(L_{3}\right)$ such that

$$
\left\|x^{\prime}\right\|_{\infty}<M_{4}
$$

From (15), (17), (20) and (23), we see that the estimates (8) hold.

Define $E=\left\{x \in W^{2, p}\left([0, T], \mathbb{R}^{n}\right): x(0)=x(T), x^{\prime}(0)=x^{\prime}(T)\right\}$, and set

$$
\Omega=\left\{x \in E: M_{2}<\|x\|_{\infty}<M_{3},\left\|x^{\prime}\right\|_{\infty}<M_{4}\right\} .
$$

Since $W^{2, p}\left([0, T], \mathbb{R}^{n}\right)$ is compactly embedded in $C^{1}$, we have $\Omega \subset C_{T}^{1}$. Now, from the above argument, we know that problem (7) has no solution on $\partial \Omega$. Hence condition (i) of Lemma 1 is satisfied.

Next, let

$$
\widetilde{F}(x)=\frac{1}{T} \int_{0}^{T}\left[e(t)-\frac{d}{d t} \operatorname{grad} F(x)-\operatorname{grad} G(x)\right] d t, \quad x \in E,
$$


that is,

$$
\widetilde{F}(x)=\frac{1}{T} \int_{0}^{T}[e(t)-\operatorname{grad} G(x)] d t, \quad x \in E .
$$

Then for any $d \in \mathbb{R}^{n} \cap E$, if $\widetilde{F}(d)=0$, we have

$$
\left.\frac{1}{T} \int_{0}^{T} \operatorname{grad} G(x)\right|_{x=d} d t=\frac{1}{T} \int_{0}^{T} e(t) d t
$$

or

$$
\left.\frac{\partial G(x)}{\partial x_{i}}\right|_{x=d}=\bar{e}_{i}, \quad i=1, \ldots, n .
$$

Hence $\left|d_{i}\right| \leq M(i=1, \ldots, n)$. Indeed, suppose that there exists $i_{0} \in$ $\{1, \ldots, n\}$ such that $\left|d_{i_{0}}\right|>M$. Then from condition $\left(\mathrm{R}_{4}\right)$, one has

$$
\left.\frac{\partial G(x)}{\partial x_{i_{0}}}\right|_{x=d} \neq \bar{e}_{i_{0}},
$$

which contradicts (24). Thus, by (16), one has $\|d\|_{\infty} \leq M<M_{3}$.

\section{Again}

$$
\partial \Omega \cap \mathbb{R}^{n}=\left\{d \in \Omega \cap \mathbb{R}^{n}:\|d\|_{\infty}=M_{2}\right\} \cup\left\{d \in \Omega \cap \mathbb{R}^{n}:\|d\|_{\infty}=M_{3}\right\} .
$$

According to the above argument, $\widetilde{F}(d) \neq 0$ for any $d \in \Omega \cap \mathbb{R}^{n}$ with $\|d\|_{\infty}=M_{3}$. Moreover, if $d \in \Omega \cap \mathbb{R}^{n},\|d\|_{\infty}=M_{2}$ and $\widetilde{F}(d)=0$, then from (19), (24), we get

$$
\begin{aligned}
M_{2} \sum_{i=1}^{n}\left|\bar{e}_{i}\right| & <\left|\left\langle d,\left.\operatorname{grad} G(x)\right|_{x=d}\right\rangle\right|=\left|\sum_{i=1}^{n} d_{i} \frac{\partial G(x)}{\partial x_{i}}\right|_{x=d} \mid \\
& =\left|\sum_{i=1}^{n} d_{i} \bar{e}_{i}\right| \leq \sum_{i=1}^{n}\left|d_{i}\right| \cdot\left|\bar{e}_{i}\right| \leq M_{2} \sum_{i=1}^{n}\left|\bar{e}_{i}\right|
\end{aligned}
$$

which is a contradiction. So $\widetilde{F}(d) \neq 0$ for all $d \in \partial \Omega \cap \mathbb{R}^{n}$, and condition (ii) of Lemma 1 holds.

Finally, we show that condition (iii) of Lemma 1 holds. Indeed, since $\widetilde{F}(d)=T^{-1} \int_{0}^{T} e(t) d t-\left.\operatorname{grad} G(x)\right|_{x=d}$ and $G \in C^{1}\left(\mathbb{R}^{n} \backslash\{0\}, \mathbb{R}\right)$, it follows that $\widetilde{F}: \Omega \cap \mathbb{R}^{n} \rightarrow \underset{\mathbb{R}^{n}}{\widetilde{F}}$ is continuous and maps bounded sets to relatively compact sets, hence $\widetilde{F}$ is completely continuous.

Set

$$
H(d, \lambda)=\lambda \Lambda(d)+(1-\lambda) \widetilde{F}(d)
$$

where $\Lambda: \mathbb{R}^{n} \rightarrow \mathbb{R}^{n}$ is the identity. Then

$$
H(d, \lambda) \neq 0, \quad \forall d \in \partial \Omega \cap \mathbb{R}^{n}, \forall \lambda \in[0,1] .
$$


In fact, for any $d \in \mathbb{R}^{n}$, if $H(d, \lambda)=0$, then $\left|d_{i}\right| \leq M(i=1, \ldots, n)$. Indeed, if $\left|\bar{d}_{i_{0}}\right|>M$ for some $\bar{d}=\left(\bar{d}_{1}, \ldots, \bar{d}_{n}\right) \in \mathbb{R}^{n}$ and $i_{0} \in\{1, \ldots, n\}$ such that $H(\bar{d}, \bar{\lambda})=0$, then

$$
\bar{\lambda} \Lambda(\bar{d})+(1-\bar{\lambda}) \widetilde{F}(\bar{d})=0,
$$

or

$$
\bar{\lambda} \bar{d}_{i}=(1-\bar{\lambda})\left(\left.\frac{\partial G(x)}{\partial x_{i}}\right|_{x=\bar{d}}-\bar{e}_{i}\right), \quad i=1, \ldots, n .
$$

In particular,

$$
\bar{\lambda} \bar{d}_{i_{0}}=(1-\bar{\lambda})\left(\left.\frac{\partial G(x)}{\partial x_{i_{0}}}\right|_{x=\bar{d}}-\bar{e}_{i_{0}}\right),
$$

so $\bar{\lambda} \neq 1$ (otherwise $\bar{d}_{i_{0}}=0$, contradicting $\left|\bar{d}_{i_{0}}\right|>M$ ). So from condition $\left(\mathrm{R}_{4}\right)$, one has

$$
\bar{d}_{i_{0}} \cdot(1-\bar{\lambda})\left(\left.\frac{\partial G(x)}{\partial x_{i_{0}}}\right|_{x=\bar{d}}-\bar{e}_{i_{0}}\right)<0,
$$

which contradicts $\lambda \bar{d}_{i_{0}}^{2} \geq 0$; thus $\left|d_{i}\right| \leq M(i=1, \ldots, n)$. Hence $\|d\|_{\infty}<$ $M<M_{3}$. So for any $d \in \Omega \cap \mathbb{R}^{n}$ with $\|d\|_{\infty}=M_{3}, H(d, \lambda) \neq 0$ for any $\lambda \in[0,1]$.

On the other hand, for any $d \in \Omega \cap \mathbb{R}^{n}$ with $\|d\|_{\infty}=M_{2}$, if $H(d, \lambda)=0$, then

$$
\lambda d+(1-\lambda)\left[\frac{1}{T} \int_{0}^{T} e(t) d t-\left.\operatorname{grad} G(x)\right|_{x=d}\right]=0,
$$

which implies

$$
\lambda\langle d, d\rangle=(1-\lambda)\left[\left\langle d,\left.\operatorname{grad} G(x)\right|_{x=d}\right\rangle-\langle d, \bar{e}\rangle\right],
$$

where

$$
\bar{e}=\frac{1}{T} \int_{0}^{T} e(t) d t .
$$

In view of $(19),\left(\mathrm{R}_{1}\right),(27)$, we have (noticing $\lambda \neq 1$ )

$$
\begin{aligned}
\lambda\langle d, d\rangle & <(1-\lambda)\left[-M_{2} \sum_{i=1}^{n}\left|\bar{e}_{i}\right|+|\langle d, \bar{e}\rangle|\right] \\
& \leq(1-\lambda)\left[-M_{2} \sum_{i=1}^{n}\left|\bar{e}_{i}\right|+\sum_{i=1}^{n}\left|d_{i}\right| \cdot\left|\bar{e}_{i}\right|\right] \\
& \leq(1-\lambda)\left[-M_{2} \sum_{i=1}^{n}\left|\bar{e}_{i}\right|+M_{2} \sum_{i=1}^{n}\left|\bar{e}_{i}\right|\right]=0,
\end{aligned}
$$

which is a contradiction. So $H(d, \lambda) \neq 0$ for any $d \in \Omega \cap \mathbb{R}^{n}$ with $\|d\|_{\infty}=M_{2}$. Hence (25) holds. 
By the homotopy property of degree

$$
\begin{aligned}
\operatorname{deg}_{\mathrm{B}}\left(\widetilde{F}, \Omega \cap \mathbb{R}^{n}, 0\right) & =\operatorname{deg}_{\mathrm{B}}\left(H(\cdot, 0), \Omega \cap \mathbb{R}^{n}, 0\right) \\
& =\operatorname{deg}_{\mathrm{B}}\left(H(\cdot, 1), \Omega \cap \mathbb{R}^{n}, 0\right) \\
& =\operatorname{deg}_{\mathrm{B}}\left(\Lambda, \Omega \cap \mathbb{R}^{n}, 0\right) \neq 0 .
\end{aligned}
$$

Thus condition (iii) of Lemma 1 holds. Hence by Lemma 1 , the periodic boundary value problem (1), (2) has at least one solution. This completes the proof.

Next, for the attractive type, using the above techniques, we can easily prove the following theorem.

TheOrem 2. Assume that conditions $\left(\mathrm{R}_{2}\right),\left(\mathrm{R}_{3}\right)$ of Theorem 1 are satisfied, and furthermore the following conditions hold:

$\left(\mathrm{R}_{1}^{\prime}\right) \quad \lim _{x \rightarrow 0}\langle x, \operatorname{grad} G(x)\rangle=\infty$.

$\left(\mathrm{R}_{4}^{\prime}\right) \quad$ There exists a constant $M>0$ such that if $\left|x_{i}\right|>M(i=1, \ldots, n)$, then

$$
x_{i}\left(\frac{\partial G}{\partial x_{i}}-\bar{e}_{i}\right)>0 \quad \text { for all } i \in\{1, \ldots, n\}
$$

where $\bar{e}_{i}=T^{-1} \int_{0}^{T} e_{i}(t) d t(i=1, \ldots, n)$.

Then the periodic boundary value problem (1), (2) has at least one solution provided

$$
a<\left(\pi_{p} / T\right)^{p}
$$

where $\pi_{p}$ is defined by (6).

\section{References}

[1] A. Ambrosetti, Critical points and nonlinear variational problems, Mem. Soc. Math. France 49 (1992).

[2] V. Coti Zelati, Dynamical systems with effective-like potentials, Nonlinear Anal. 12 (1988), 209-222.

[3] W. B. Gordon, Conservative dynamical systems involving strong forces, Trans. Amer. Math. Soc. 204 (1975), 113-135.

[4] P. Habets and L. Sanchez, Periodic solutions of dissipative dynamical systems with singular potentials, Differential Integral Equations 3 (1990), 1139-1149.

[5] -, -, Periodic solutions of some Liénard equations with singularities, Proc. Amer. Math. Soc. 109 (1990), 1035-1044.

[6] A. C. Lazer and S. Solimini, On periodic solutions of nonlinear differential equations with singularities, ibid. 99 (1987), 109-114.

[7] R. Manásevich and J. Mawhin, Periodic solutions for nonlinear systems with p-Laplacian-like operators, J. Differential Equations 145 (1998), 367-393.

[8] J. Mawhin, Topological degree and boundary value problems for nonlinear differential equations, in: Topological Methods for Ordinary Differential Equations, M. Furi and P. Zecca (eds.), Lecture Notes in Math. 1537, Springer, New York, 1993, 74-142. 
[9] S. Solimini, On forced dynamical systems with a singularity of repulsive type, Nonlinear Anal. 14 (1990), 489-500.

[10] M. R. Zhang, Periodic solutions of damped differential systems with repulsive singular forces, Proc. Amer. Math. Soc. 127 (1999), 401-407.

[11] - Nonuniform nonresonance at the first eigenvalue of the p-Laplacian, Nonlinear Anal. 29 (1997), 41-51.

[12] - - A relationship between the periodic and the Dirichlet BVPs of singular differential equations, Proc. Roy. Soc. Edinburgh Sect. A 128 (1998), 1099-1114.

Department of Mathematics

Huazhong University of Science and Technology

Wuhan 430074, P.R. China

E-mail: bingliug@public.wh.hb.cn
College of Mathematics and Statistics

Wuhan University Wuhan 430072, P.R. China

Reçu par la Rédaction le 15.11.2000

Révisé le 29.3.2001 et 5.3.2002 